\title{
Policy on Downlink Access of Femtocell Based on OFDMA in Dense Employment Residential Environment
}

\author{
Huanle Zhang $^{1, \text { a }}$ and Jian Liu $^{2, b}$ \\ ${ }^{1}$ University of Electronic Science and Technology of China, Chengdu, P. R. China \\ ${ }^{2}$ University of Science and Technology Beijing, Beijing, P. R. China. \\ azhanghuanle1342@gmail.com, bianliusdu@gmail.com
}

Keywords: Downlink Access, Femtocell, OFDMA, Data Rate, Outage Probability.

\begin{abstract}
The femtocell technology has spurred much interest both in research field and industrial area. There would be a widespread employment of femtocell access points (FAPs) in residential environment in near future. However, in a typical residential buildings with two rows of apartments which have femtocell access points (FAPs) installed and a long and narrow corridor in between, the Signal to Interference Plus Noise Ratio (SINR) of macrocell signal for passengers would be degraded dramatically that practical communicaitons would be impossible. It seems that access to nearby FAPs would be the only plausible solution. However, access to the strongest pilot FAP maybe inefficient in this dense FAPs employment environment. In this thesis, comparisons between different downlink access methods for FAPs based on OFDMA in dense employment residential environment have been made and their respective data rate and outage probability have been achieved.
\end{abstract}

\section{Introduction}

Femtocell interest in the mobile operator community continues to grow, deployments increased to 41 in 23 countries during 2012[1]. It is estimated by Informa Telecoms \& Media that the deployments of small cell market would reach 91.9 million by 2016, with femtocells accounting for more than $80 \%$. It is expected the femtocell access points (FAPs) would be widespread employed in near future. However, in a typical residential buildings with two rows of apartments which have femtocell access points (FAPs) installed and a long and narrow corridor in between, the Signal to Interference Plus Noise Ratio (SINR) of macrocell signal for passengers would be degraded dramatically that practical communications would be impossible. It seems that access to nearby FAPs would be the only reasonable solution.

However, the owner of the FAP may be reluctant to accept the access of passengers because of the concerns about its communication quality degraded, so the attachment of passengers should have no or little impact on the owner's performance. In addition, the access of passengers to the strongest pilot FAP may be inefficient in this dense employment environment. For example, the FAP which the passengers attached to could have no available resources or the resources could be interfered strongly that result in a terrible experience of the passengers, whereas other FAPs may provider more pleasing experience to passengers even though their pilot strength is weaker than the preceding FAP. Therefore, more efficient access methods should be investigated rather than the always access to the strongest pilot FAP method. Nevertheless, adaptive algorithms may not be suitable owe to its complex and power-hungry property that is intolerable to the simple mobile terminals.

In this article ${ }^{1}$, we propose some simple but realistic access algorithms and compare their data rate and outage probability. We are focus on the FAPs based on the LTE/OFDMA technology, but it also applicable to other standards in similar environment, such as WiMAX which also adopt OFDMA. The article is organized as follows: First, we give brief but relevant information on the downlink access of FAPs based on LTE/OFDMA; second, our simulation model is proposed and results are analyzed; at last, we summarize the article.

1 This work is supported by the National Natural Science Foundation of China (nos. 60932002 and 61173149), the National Major Projects (nos. 2012ZX03001029-005 and 2012ZX03001032-003), and the Fundamental Research Funds for the Central Universities (no. FRF-TP-12-080A). 


\section{Preliminaries}

The simulation scenario is depicted in Fig. 1. In each room, a number of resources have already allocated to the owners of the FAPs. Meanwhile, a passenger is passing through the corridor at a constant walking velocity, requesting access to the FAPs. The passenger should not contend for the resources with the owners. Only the unused resources may be assigned to the passenger. In this article, we want to explore the data rate and outage probability he can achieve under different downlink access algorithms. Details will be given in following sections.

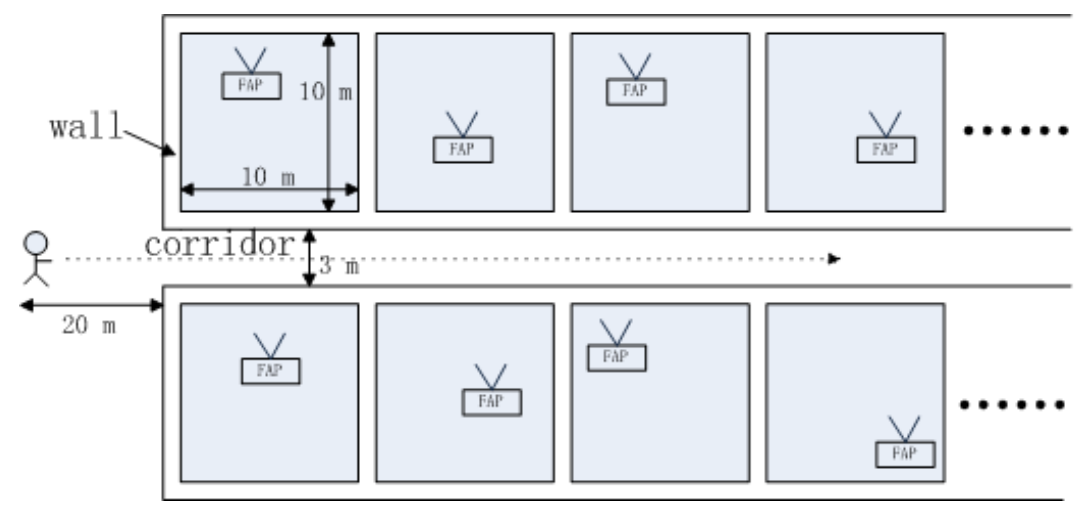

Fig. 1. system simulation model

RF Propagation Model. The propagation effects of RF can be divided into three distinct types, i.e., mean path loss, slow variation about the mean due to scattering and shadowing, and the rapid variation in signal due to multipath effects. The ITU-R 3G indoor office test channel models (ITU-R M.1225) [5] is adopted in our simulation and our arguments are set as below.

The indoor path lose model $(\mathrm{dB})$ is described in [5] as:

$$
L=37+40 \lg (R)+18.3 n^{\left(\frac{n+2}{n+1}-0.46\right)}
$$

where, $R$ is the transmitter-receiver separation (m) and $\mathrm{n}$ is the number of floors in the path.

Since we are only focus on the same floor where the passenger pass through the corridor (as Fig. 1 shows), there are no path loss of floor are considered. $15 \mathrm{~dB}$ attenuation is added for each wall as the RF penetrates from the FAP to the passenger. Furthermore, a log-normal shadow fading standard deviation of $12 \mathrm{~dB}$ can be expected in this test model [5]. So the resulting indoor path lose model will be:

$$
L=37+40 \lg (R)+15 n+12
$$

where, $n$ is the number of walls in the path in this equation.

In addition to the above path lose; there are also multipath components that should be considered.

Downlink Resource Structure. The downlink transmission resources in LTE possess dimensions of time, frequency and space. The time-frequency reources are subdivided according to the following structure [3]: the largest unit of time is the $10 \mathrm{~ms}$ radio frame, which is further divided into ten $1 \mathrm{~ms}$ subframes, each of which is split into two $0.5 \mathrm{~ms}$ slots. Each slot consists of seven OFDM symbols in the case of the normal or six if the extended cyclic prefix is configured.

A minimum of 72 subcarriers must be supported, and 12 subcarriers (occupying a total of $180 \mathrm{kHz}$ ) are grouped into a subchannel. In our article, the minimum of 6 subchannels are assumed for each FAP. The passenger is allowed to access to a subchannel only if the subchannel is unused at the FAP he attached to.

Data Rate. For simplicity, we assume Shannon Channel Capacity formula as a metric for our data rate comparison, that is, $C=W \log (1+S / S I N R)$. Since the passenger only access to a subchannel, we take $W=180 \mathrm{kHz}$. The signal power here means the receive power from the FAP which the passenger 
attached to in the particular subchannel, while the SINR means other power received, that is, interference and thermal noise.

Outage Probability. The probability of the event is called the outage probability if $P_{\text {out }}(R)=P[C$ $<R]$. For a Rayleigh fading channel with normalized channel gain, we have $C=W \log (1+\rho \operatorname{SINR})$, where $\rho$ is an exponential random variable with expected value equal to 1[6]. In this case, the outage probability is simplified to

$$
P_{\text {out }}(R)=P\left[\rho<\frac{2^{\frac{R}{W}}-1}{\operatorname{SINR}}\right]=1-\exp \left(-\frac{2^{\frac{R}{W}}-1}{\operatorname{SINR}}\right)
$$

\section{System Model and Results Analysis}

This section will give more detail about our simulation model and the results will be analyzed.

Simulation Model. Our simulation scenario is depicted in Fig. 1. Each room is of area $10 \mathrm{~m} \times 10 \mathrm{~m}$. There are walls between each adjacent room and between room and corridor. We take $15 \mathrm{~dB}$ attenuations for signal penetrating through each wall. For emulating the dense employment of FAPs that will take place when widespread of FAPs become available in residential environment, each room has a FAP that locates randomly following 2-D uniform distribution. The power of each FAP is $0.1 \mathrm{~W}$, uniformly distributing among each subchannel. We also assume that each FAP transmit the same strength of pilot. Furthermore, the number of subchannels already occupied by the owner of FAP is following exponential distribution with mean $N(N=1,2, \ldots, 6)$ while a remain unused subchannel can be assigned to the passenger. The passenger is walking at a constant speed, starting from $20 \mathrm{~m}$ distance from left, walking into the corridor (3 $\mathrm{m}$ width) straight towards right. Other parameters are set as in the preceding sections. We want to explore the data rate and outage probability the passenger can achieve using following access policy:

1) Access to the FAP with the most strength pilot received, and randomly occupies an unused subchannel.

2) Access to the FAP with the most strength pilot received but occupies an unused subchannle that has the highest SINR.

3) Scan for two FAPs that have the most strength pilot and occupy an unused subchannel based on the highest SINR.

4) Scan for three FAPs that have the most strength pilot and occupy an unused subchannel based on the highest SINR.

Results Analysis. As the Fig. 2 shows, the simple access method just based on pilot strength and randomly occupies a subchannel results in a low data rate compared with other methods. Even if the passenger mobile terminal just access to the most strength pilot FAP, there would be a nearly two-fold increase in data rate if the terminal scans for the highest SINR subchannel. Specifically, when the traffic is already heavy, as in the case of $\mathrm{N}=6$, there has an increase data rate of $127 \%$ for the method 2.

In the case of method 4 (scan three FAPs for the highest SINR subchannel), there is just a maximum of $13 \%$ increase in data rate compared with the method 3 . It shows that scanning three FAPs are not reasonable, accounting for the much more implement overhead and little extra data rate achieved.

In indoor environment, there is plenty of fading affecting the outage. Fig. 3 illustrates the theoretical outage probability for each access method when $\mathrm{N}=6$. It is corresponds to the data rate figure shown in Fig. 2. Above all, the method 2 and 3 seems plausible in both complexity and data rate achieved. However, method 2 results in a much higher outage probability. For instances, if the FAP is already saturated, the access request will be rejected; or if the available subchannel is interfered dramatically, the quality of communication will be terrible for the passenger. Consequently, the method of scanning two strongest pilot FAPs and access to the highest SINR subchannel would be the best choice in our employment environment. 


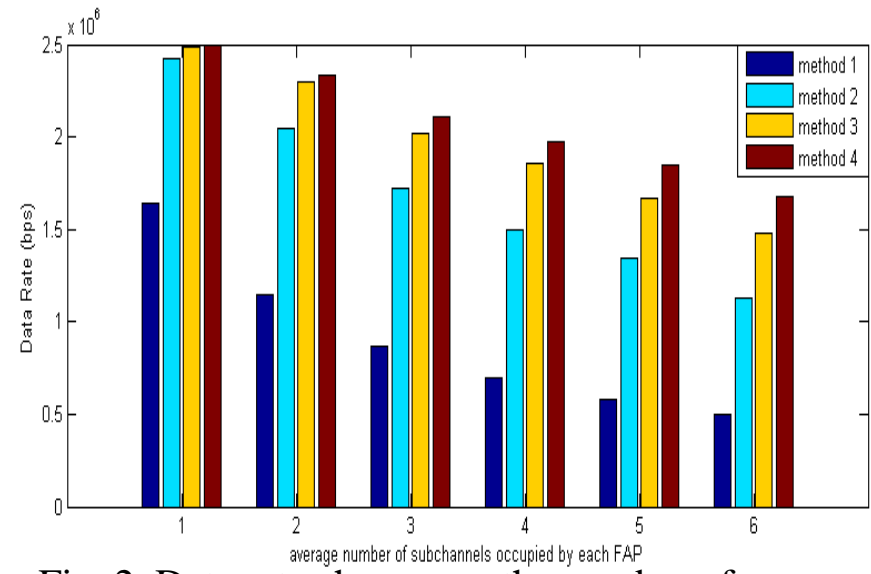

Fig. 2. Data rate change vs. the number of average subchannels already occupied in four different access methods.

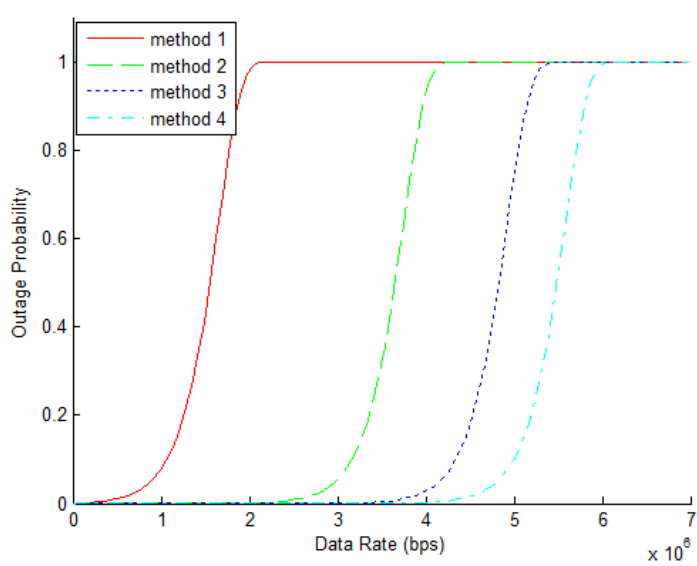

Fig. 3. Outage Probability for each access method when $\mathrm{N}=6$.

\section{Summary}

In this article, we present four different access methods for femtocell downlink access and compare each data rate and outage probability. It is clear that simply search for the most strength pilot FAP and occupy a random unused subchannel will result in low data rate and high outage probability. Through the comparison, we draw the conclusion that in dense employment of FAPs like ours, search for the two strongest pilot FAPs and access to the highest SINR subchannel will be preferred in terms of data rate, outage probability and implement overhead. With the rapid development of femtocell market, dense employment like the scenario we propose will occur. Thus a comprehensive effect on downlink access performance should be explored.

\section{References}

[1] Small Cell Forum, http://www.smallcellforum.org/, 2012.

[2] "Wifi and femtocell integration strategies 2011-2015," Juniper Research Whitepaper, http://www.juniperresearch.com/, Mar. 2011.

[3] J. G. Andrews, H. Claussen, M. Dohler, S. Rangan, and M. C. Reed, "Femtocells: Past, Present, and Future," IEEE Journal on Selected Areas in Communications, vol. 30, no. 3, Apr. 2012.

[4] H.-S. Jo, Y. J. Sang, P. Xia, and J. G. Andrews, "Outage probability for heterogeneous cellular networks with biased cell association," IEEE Globecom, Dec. 2011.

[5] Recomemdation ITU-R M.1225, Guidelines for evaluation of radio transmission technologies for IMT-2000, 1997.

[6] John G. Proakis, Digital Communications, Fifth Edition , pp. 915, Publishing House of

Electronics Industy, 2009. 